\title{
Correlation between preoperative pressure pain assessments and anxiety and postoperative pain in impacted lower third molar surgery
}

\author{
Hatice Hosgor, Fatih Mehmet Coskunses, Berkay Tokuc \\ Department of Oral and Maxillofacial Surgery, Faculty of Dentistry, Kocaeli University, Kocaeli, Turkey
}

\begin{abstract}
J Korean Assoc Oral Maxillofac Surg 2021;47:15-19)
Objectives: The aim of this study was to evaluate correlations between anxiety and preoperative pressure pain assessments and postoperative pain and analgesic requirements in impacted lower third molar tooth surgery.

Materials and Methods: This prospective study enrolled 60 patients who underwent impacted lower third molar surgery. The preoperative StateTrait Anxiety Inventory-I (STAI-I), pressure pain threshold, and pressure pain tolerance scores were measured. At 2, 4, 6, 12, and 24 hours, and at 6 days following surgery, the patients scored their pain on the visual analogue scale and recorded their analgesic drug usage. The data were evaluated, and the results were statistically analyzed.

Results: Of the 60 patients, 38 were female. Mean age was $24.62 \pm 7.42$ years. The study found no relationship between preoperative pressure pain assessments and postoperative pain $(P>0.05)$. There was also no relationship observed between preoperative STAI-I scores and postoperative pain $(P>0.05)$. However, there was a positive correlation between operation time and total medication taken $(P<0.05)$.

Conclusion: Preoperative pressure pain threshold, pressure pain tolerance, and anxiety level had no significant effects on postoperative pain and analgesic requirements in impacted lower third molar surgery.
\end{abstract}

Key words: Pain assessment, Anxiety, Postoperative pain, Third molar

[paper submitted 2020. 6. 23 / revised 1st 2020. 9. 3, 2nd 2020. 9. 4 / accepted 2020. 9. 29]

\section{Introduction}

The removal of impacted third molar teeth is one of the most common oral surgical procedures ${ }^{1}$. Planning and surgical skills are critical during preoperative diagnosis and postoperative management ${ }^{2}$. Postoperative pain is a well-known phenomenon that commonly occurs after almost all oral and maxillofacial surgeries ${ }^{3}$. In the early postoperative period, pain reaches its maximum intensity, and analgesics are often required $^{4}$.

The pain threshold is the most variable physical marker for pain. The most convenient measurement method is algom-

\author{
Hatice Hosgor \\ Department of Oral and Maxillofacial Surgery, Faculty of Dentistry, \\ Kocaeli University, Basiskele, Kocaeli 41190, Turkey \\ TEL: +90-530-252-46-24 \\ E-mail:drhaticehosgor@yahoo.com \\ ORCID: https://orcid.org/0000-0002-6925-9526
}

(2) This is an open-access article distributed under the terms of the Creative Commons Attribution Non-Commercial License (http://creativecommons.org/ licenses/by-nc/4.0/), which permits unrestricted non-commercial use, distribution and reproduction in any medium, provided the original work is properly cited.

Copyright (C) 2021 The Korean Association of Oral and Maxillofacial Surgeons. All rights reserved. etry ${ }^{5}$. Emotional factors could affect a patient's pain threshold $^{6}$. An association has been found between preoperative anxiety and postoperative pain ${ }^{7}$. Preoperative anxiety may increase the subjective experience of pain, and this may lead to increased analgesic use and a prolonged hospital stay ${ }^{8}$. The State-Trait Anxiety Inventory (STAI) scale is the most commonly used test for measuring anxiety ${ }^{9}$. Current studies suggest that postoperative pain and analgesic requirements may be affected by factors such as surgery type, anxiety, depression, and preoperative pain ${ }^{10,11}$. The correlation between the pressure pain threshold and tolerance and postoperative pain has been evaluated in several branches of medicine $e^{12-14}$; however, few studies on oral and maxillofacial surgery have included these parameters. A review of the literature indicates that the current study is the first to do so.

The primary aim of this study was to investigate the effects of anxiety and preoperative pressure pain threshold and tolerance on postoperative pain and analgesic requirements in impacted lower third molar surgery. The secondary aim was to evaluate the effects of tooth retention, operation time, and previous surgical experience. 


\section{Materials and Methods}

The research protocol was approved by the Clinical Research Ethics Committee of Kocaeli University (KÜ GOKAEK 2019/15), and written informed consent was obtained from all patients. The study followed the Declaration of Helsinki guidelines on medical ethics and protocols. This prospective study evaluated data obtained from patients who were seen at Faculty of Dentistry, Kocaeli University from January to April 2019 and required to undergo the extraction of impacted lower third molar teeth prophylactically.

A total of 60 patients who underwent impacted lower third molar surgeries were included in the study after their written consents were obtained. The inclusion criteria were the absence of: (1) alcohol or drug addiction, (2) analgesic drug use, and (3) chronic pain. Patients with medical problems that could pose limitations in the evaluation were excluded from the study. Infected and painful teeth were not included.

Preoperative anxiety and the pressure pain threshold and tolerance were measured. Anxiety was assessed using the STAI-I, and the pressure pain threshold and tolerance were measured using an algometer with a $1 \mathrm{~cm}^{2}$ probe tip (Baseline Dolorimeter, 22-lb gauge; Fabrication Enterprises, White Plains, NY, USA). The measurement site was the tip of the third finger on the right hand. Before the measurements were taken, the patients were fully informed of the research and measurement procedures. For the pressure pain threshold measurement, the patients were asked to say "stop" at the pressure value at which they first felt pain. For the pressure pain tolerance measurement, the patients were asked to say "stop" at the maximum pressure value at which they could voluntarily withstand the pain. All measurements were repeated three times at 10-minute intervals. The pressure pain thresholds and tolerances were derived from the average values of the three measurements. All measurements were made by the same physician using the same equipment in the same room at the same room temperature. The pressure pain threshold and tolerance were categorized as low and high. Patients with values below each group mean were coded as "low", and those with values above the mean were coded as "high".

Age, sex, previous surgical experience, and tooth retention status (mucosal or bone) were recorded for each patient. In this study, all teeth with mucosal and bone retention were in a vertical position. Only one tooth was extracted from each patient. The impacted lower third molar surgeries were performed by one surgeon.
All operations were performed under local anesthesia in the operating room of Department of Oral and Maxillofacial Surgery, Faculty of Dentistry, Kocaeli University. Appropriate asepsis and antisepsis precautions were taken. The mucoperiosteal flap was removed within the classic framework for the extraction of impacted lower third molars. When necessary, the bone was drilled under saline washing, and tooth extraction was performed. Following bleeding control, the wound was sutured as primary. The time from the application of local anesthesia to the end of the suturing procedure was calculated and recorded as the operation time. In the postoperative period, the patients were prescribed antibiotics, analgesics, and antiseptic mouthwashes. The sutures were removed on the seventh postoperative day. Each patient received a naproxen sodium $550 \mathrm{mg}$ tablet (Apranax Fort; Abdi Ibrahim Ilac, Istanbul, Turkey) prior to the surgical procedure. The same drug was prescribed after surgery. The patients were asked to note the number of painkillers they received during the postoperative period. A visual analogue scale (VAS) with values ranging from 0 to 10 was used to evaluate postoperative pain. On a form that they were provided, the patients were asked to record their VAS scores at 2, 4, 6, 12, and 24 hours and during the 6 days following surgery. They were asked to bring this form when they returned for suture removal the following week. From these data, the total number of medications (number of tablets) taken by each patient was calculated.

Statistical analyses were performed with SPSS for Windows (ver. 16; SPSS, Chicago, IL, USA). The Shapiro-Wilk test was used to test normality of the parameters. Descriptive statistics (mean, standard deviation, frequencies, and percentage) were used to evaluate the data. For the quantitative data, analysis of variance was used to compare the normally distributed parameters, and the Student's $t$-test was used to compare two groups. Kruskal-Wallis and Mann-Whitney U tests were used to compare the non-normally distributed parameters of the quantitative data. Pearson's correlation coefficient was used to compare the correlations in the normally distributed quantitative data, and Spearman's rho was used to compare the correlations in the non-normally distributed data. The confidence interval was $95 \%$, and statistical significance was set as $P<0.05$.

\section{Results}

A total of 60 patients were included in this study. The ages ranged from 16 to 49 , with a mean age of $24.62 \pm 7.42$ years. 
Table 1. Demographic data distribution

\begin{tabular}{ll}
\hline \multicolumn{1}{c}{ Variable } & Value \\
\hline Sex & $38(63.3)$ \\
Female & $22(36.7)$ \\
Male & \\
Previous surgical experience & $21(35.0)$ \\
Yes & $39(65.0)$ \\
No & \\
Tooth retention & $19(31.7)$ \\
Mucosal retention & $41(68.3)$ \\
Bone retention & \\
Pressure pain threshold & $27(45.0)$ \\
Low & $33(55.0)$ \\
High & \\
Pressure pain tolerance & $29(48.3)$ \\
Low & $31(51.7)$ \\
High &
\end{tabular}

Values are presented as number (\%).

Hatice Hosgor et al: Correlation between preoperative pressure pain assessments and anxiety and postoperative pain in impacted lower third molar surgery. J Korean Assoc Oral Maxillofac Surg 2021

Patient demographics are presented in Table 1, and the average, minimum, and maximum values of the demographic data are provided in Table 2.

No significant relationships were found between the VAS scores at 2, 4, 6, 12, and 24 hours and during the 6 days following surgery. There was also no significant relationship between the low and high pressure pain threshold and pressure pain tolerance values $(P>0.05)$. No significant relationships were found between the number of analgesic drugs used on any of the first seven days after surgery and the low and high pressure pain threshold and pressure pain tolerance values $(P>0.05)$. There were significant positive correlations between the VAS scores on each of the first seven days after surgery and the number of analgesic drugs used $(P<0.05)$.

There was a positive correlation between operation time and total number of medications used $(r=0.276 ; P=0.033)$. However, there were no significant correlations among pressure pain threshold, pressure pain tolerance, STAI-I score, tooth retention status, previous surgical experience, and total number of medications taken $(P>0.05)$. The STAI-I scores for patients with high pressure pain tolerance values were significantly higher than those for patients with low values $(P=0.036)$. However, there was no significant correlation between the STAI-I score and the pressure pain threshold $(P>0.05)$.

There were no significant differences between previous surgical experience and STAI-I score, pressure pain threshold, or pressure pain tolerance $(P>0.05)$.
Table 2. Descriptive statistics of demographic data

\begin{tabular}{lc}
\hline \multicolumn{1}{c}{ Variable } & Value \\
\hline Age (yr) & $24.62 \pm 7.42(16-49)$ \\
Pressure pain threshold (lbs) & $12.37 \pm 3.55(5.50-18.75)$ \\
Pressure pain tolerance (lbs) & $16.79 \pm 4.01(9.25-22.00)$ \\
STAI-I score & $40.53 \pm 7.51(25-70)$ \\
Total analgesic drug (No. of tablets) & $6.52 \pm 3.48(0-18)$ \\
\hline
\end{tabular}

(STAI-I: State-Trait Anxiety Inventory-I)

Values are presented as mean \pm standard deviation (range).

Hatice Hosgor et al: Correlation between preoperative pressure pain assessments and anxiety and postoperative pain in impacted lower third molar surgery. J Korean Assoc Oral Maxillofac Surg 2021

\section{Discussion}

A review of the literature revealed studies in which correlations with postoperative pain were determined through evaluations of patients' preoperative physical and psychological characteristics $^{12,15}$. In a study similar to the current study, Hsu et al. ${ }^{12}$ evaluated the correlations between the preoperative pressure pain threshold and tolerance and postoperative pain and total analgesics taken after lower abdominal gynecologic surgery. They found no correlations between the pressure pain threshold and tolerance and postoperative VAS scores; however, postoperative morphine use decreased as the pressure pain tolerance increased. Duan et al. ${ }^{14}$ found a correlation between higher pain tolerance and the decreased use of postoperative analgesia in patients who underwent elective surgery under general anesthesia. However, a correlation between the pressure pain threshold and postoperative analgesia was not reported.

The widely accepted conclusions from comparisons of the efficacy of the preoperative pressure pain threshold and preoperative pain tolerance suggest that pain tolerance is a better predictor of postoperative pain ${ }^{12,16}$. The present study found no correlations between the preoperative pressure pain threshold and tolerance and postoperative pain or total analgesic use. The literature indicates that studies have focused mostly on major surgical procedures requiring general anesthesia, thereby leading to higher VAS scores and higher analgesic needs. The lack of statistical significance in the current study could be attributed to the use of local anesthesia. The procedures were less traumatic than those in previous studies.

Preoperative anxiety plays a critical role in the chain of events that affect postoperative pain management ${ }^{8}$. Anxiety has also been reported to be a factor in reducing pain threshold $^{17}$. The current study found that pressure pain tolerance decreased as anxiety increased. Previous studies have found a positive correlation between postoperative pain and anxi- 
ety. Moreover, increased anxiety and fear have been found to lead to greater pain severity, thereby increasing postoperative analgesic requirements. This could in turn lead to excessive medication use to overcome stress and anxiety ${ }^{18}$. The current study found no association between anxiety and the total medication used.

The conclusions concerning the correlation between anxiety and previous surgical experience are contradictory. Kaakko and Murtomaa ${ }^{19}$ revealed that patients with previous experience with tooth extraction had higher pre-surgery anxiety. In contrast, another study found higher anxiety among patients with no previous surgical experience ${ }^{20}$. The current study found no significant difference between the patients with and without previous surgical experience regarding the pressure pain threshold and tolerance, STAI-I scores, and total medication used.

Postoperative pain is associated with surgical factors, such as the surgical site, difficulty, and technique ${ }^{21}$. The general opinion in the literature is that postoperative pain and analgesic use are positively associated with surgical difficulty ${ }^{22,23}$. Lago-Méndez et al. ${ }^{22}$ found a correlation between surgical difficulty and postoperative pain in impacted mandibular third molar extractions. Longer operation time led to greater postoperative pain. The current study found no difference in the pain and analgesic requirements after the extraction of teeth with bone and mucosal retention. However, as expected, a positive correlation was found between operation time and analgesic use.

\section{Conclusion}

The primary conclusion of this study is that the preoperative pressure pain threshold, pressure pain tolerance, and anxiety level had no significant effects on postoperative pain and analgesic requirements in impacted lower third molar surgery. A secondary finding was the association between operation time and postoperative analgesic use.

\section{ORCID}

Hatice Hosgor, https://orcid.org/0000-0002-6925-9526

Fatih Mehmet Coskunses, https://orcid.org/0000-00018764-5992

Berkay Tokuc, https://orcid.org/0000-0002-1149-2886

\section{Authors' Contributions}

H.H. was responsible for the conception and design of the study and for data collection. F.M.C. and B.T. contributed to data analysis and interpretation, drafting the article, and critical revision of the article.

\section{Ethics Approval and Consent to Participate}

The study was approved by the Clinical Research Ethics Committee of Kocaeli University (KÜ GOKAEK 2019/15), and written informed consent was obtained from all patients.

\section{Conflict of Interest}

No potential conflict of interest relevant to this article was reported.

\section{References}

1. Park W, Kim JH, Kang SH, Kim MK, Kim BC, Choi JW, et al. Reconsideration of decision making for third molar extraction. J Korean Assoc Oral Maxillofac Surg 2011;37:343-8. https://doi. org/10.5125/jkaoms.2011.37.5.343

2. Susarla SM, Dodson TB. Risk factors for third molar extraction difficulty. J Oral Maxillofac Surg 2004;62:1363-71. https://doi. org/10.1016/j.joms.2004.05.214

3. Cillo JE. Peri-operative pain management in maxillofacial surgery. In: Ferneini E, Bennett J, eds. Perioperative assessment of the maxillofacial surgery patient: problem-based patient management. Cham: Springer; 2018:145-60.

4. Comfort MB, Tse AS, Tsang AC, McGrath C. A study of the comparative efficacy of three common analgesics in the control of pain after third molar surgery under local anaesthesia. Aust Dent J 2002;47:327-30. https://doi.org/10.1111/j.1834-7819.2002. tb00546.x

5. Koo TK, Guo JY, Brown CM. Test-retest reliability, repeatability, and sensitivity of an automated deformation-controlled indentation on pressure pain threshold measurement. J Manipulative Physiol Ther 2013;36:84-90. https://doi.org/10.1016/j.jmpt.2013.01.001

6. Martinez-Calderon J, Meeus M, Struyf F, Diaz-Cerrillo JL, Clavero-Cano S, Morales-Asencio JM, et al. Psychological factors are associated with local and generalized pressure pain hypersensitivity, pain intensity, and function in people with chronic shoulder pain: a cross-sectional study. Musculoskelet Sci Pract 2019;44:102064. https://doi.org/10.1016/j.msksp.2019.102064

7. Ciccozzi A, Marinangeli F, Colangeli A, Antonacci S, Pilerci G, Di Stefano L, et al. Anxiolysis and postoperative pain in patients undergoing spinal anesthesia for abdominal hysterectomy. Minerva Anestesiol 2007;73:387-93.

8. Kain ZN, Sevarino F, Pincus S, Alexander GM, Wang SM, Ayoub $\mathrm{C}$, et al. Attenuation of the preoperative stress response with midazolam: effects on postoperative outcomes. Anesthesiology 2000;93:141-7. https://doi.org/10.1097/00000542-20000700000024

9. Spielberger CD, Gorsuch RL, Lushene R, Vagg PR, Jacobs G. Manual for the state-trait anxiety inventory. Palo Alto: Consulting Psychologists Press; 1983. 
10. Ip HY, Abrishami A, Peng PW, Wong J, Chung F. Predictors of postoperative pain and analgesic consumption: a qualitative systematic review. Anesthesiology 2009;111:657-77. https://doi. org/10.1097/ALN.0b013e3181aae87a

11. Astramskaite I, Juodžbalys G. Scales used to rate adult patients' psycho-emotional status in tooth extraction procedures: a systematic review. Int J Oral Maxillofac Surg 2017;46:886-98. https://doi. org/10.1016/j.ijom.2017.03.015

12. Hsu YW, Somma J, Hung YC, Tsai PS, Yang CH, Chen CC. Predicting postoperative pain by preoperative pressure pain assessment. Anesthesiology 2005;103:613-8. https://doi. org/10.1097/00000542-200509000-00026

13. Kirdemir P, Özorak Ö. Can postoperative pain and analgesic need be predicted in preoperative period? Turkiye Klinikleri J Med Sci 2011;31:951-9. https://doi.org/10.5336/medsci.2010-19299

14. Duan G, Guo S, Zhang Y, Ying Y, Huang P, Zhang L, et al. Effects of epidemiological factors and pressure pain measurements in predicting postoperative pain: a prospective survey of 1,002 Chinese patients. Pain Physician 2017;20:E903-14.

15. Werner MU, Duun P, Kehlet H. Prediction of postoperative pain by preoperative nociceptive responses to heat stimulation. Anesthesiology 2004;100:115-9; discussion 5A. https://doi. org/10.1097/00000542-200401000-00020

16. Chapman CR, Casey KL, Dubner R, Foley KM, Gracely RH, Reading AE. Pain measurement: an overview. Pain 1985;22:1-31. https://doi.org/10.1016/0304-3959(85)90145-9

17. Auerbach SM, Spielberger CD. The assessment of state and trait anxiety with the Rorschach test. J Pers Assess 1972;36:314-35. https://doi.org/10.1080/00223891.1972.10119767

18. Rawal N. Postoperatif ağrı tedavisi [Postoperative pain treatment]. In: Erdine S, ed. Ağr1 [Pain]. 1st ed. İstanbul: Nobel Tıp; 2000:124-
41. Turkish.

19. Kaakko T, Murtomaa H. Factors predictive of anxiety before oral surgery: efficacy of various subject screening measures. Anesth Prog 1999;46:3-9.

20. Matthias AT, Samarasekera DN. Preoperative anxiety in surgical patients - experience of a single unit. Acta Anaesthesiol Taiwan 2012;50:3-6. https://doi.org/10.1016/j.aat.2012.02.004

21. Gerbershagen HJ, Pogatzki-Zahn E, Aduckathil S, Peelen LM, Kappen TH, van Wijck AJ, et al. Procedure-specific risk factor analysis for the development of severe postoperative pain. Anesthesiology 2014;120:1237-45. https://doi.org/10.1097/ ALN.0000000000000108

22. Lago-Méndez L, Diniz-Freitas M, Senra-Rivera C, Gude-Sampedro F, Gándara Rey JM, García-García A. Relationships between surgical difficulty and postoperative pain in lower third molar extractions. J Oral Maxillofac Surg 2007;65:979-83. https://doi. org/10.1016/j.joms.2006.06.281

23. Negreiros RM, Biazevic MG, Jorge WA, Michel-Crosato E. Relationship between oral health-related quality of life and the position of the lower third molar: postoperative follow-up. J Oral Maxillofac Surg 2012;70:779-86. https://doi.org/10.1016/j.joms.2011.09.034

How to cite this article: Hosgor H, Coskunses FM, Tokuc B. Correlation between preoperative pressure pain assessments and anxiety and postoperative pain in impacted lower third molar surgery. J Korean Assoc Oral Maxillofac Surg 2021;47:15-19. https://doi. org/10.5125/jkaoms.2021.47.1.15 